\title{
Effect of hydrate anti-agglomerants on water-in-crude oil emulsion stability
}

\author{
Azlinda Azizi ${ }^{1}$ (D) Michael L. Johns ${ }^{1} \cdot$ Zachary M. Aman $^{1} \cdot$ Eric F. May ${ }^{1}$ Nicholas N. A. Ling ${ }^{1} \cdot$ Hazlina Husin $^{2}$
}

Received: 2 November 2018 / Accepted: 11 May 2019 / Published online: 23 May 2019

(c) The Author(s) 2019

\begin{abstract}
Under high-pressure and low-temperature conditions, gas hydrate shells may form and grow at the interface of water droplets in water-in-oil emulsions. Such hydrate formation can enable downstream agglomeration and slurry viscosification, thus increasing the risk of hydrate blockage. Therefore, emulsion stability represents a critical parameter in understanding this overall flow behaviour. In this study, the impact of three common and widely-used industrial anti-agglomerants from three different suppliers (AA-1, AA-2 and AA-3 - exact composition is commercially sensitive) on $30 \mathrm{wt} \%$ water-in-oil (W/O) emulsion stability was investigated. Bench-top nuclear magnetic resonance (NMR) pulsed field gradient (PFG) methods were used to measure the droplet size distributions (DSDs) of the W/O emulsions as a complement to bottle stability test. In the absence of hydrate anti-agglomerants, based on visual observation, $85 \%$ of the original W/O emulsion remained after $10 \mathrm{~h}$. In the presence of AA- 1 and AA-2, 94\% of the original emulsion was retained; in contrast, AA-3 acted to destabilise the emulsion with only $64 \%$ of the original emulsion visually evident after $10 \mathrm{~h}$. These results were substantiated by PFG NMR measurements which showed substantial changes in droplet size as a function of sample height for the W/O emulsion formulated with AA-3. Interestingly the W/O emulsion formulated with AA-1, while very stable, was characterised by comparatively very large water droplets, indicative of a complex multiple water-in-oil-in-water (W/O/W) emulsion microstructure. AA-2 forms stable emulsion with small droplets of water dispersed in the oil phase. Our results provide insight into a wide range of potential impacts of AA addition on an industrial crude oil pipeline, in which AA-1 resulted in a complex W/O/W multiple emulsion, AA-2 behaved as an emulsifier and AA-3 behaved as a demulsifier.
\end{abstract}

Keywords Water-in-oil emulsion $\cdot$ Hydrate anti-agglomerants $\cdot$ Nuclear magnetic resonance $\cdot$ Surface-active agents

\section{Introduction}

During oil transportation, mixing between viscous crude oil and any aqueous phase present can lead to the formation of water-in-oil (W/O) emulsions, especially in high-shearrate conditions. Such emulsions contain both dispersed and continuous phases and can be stabilised by surfactants and/ or solid particles. In oilfield pipelines where W/O emulsions are commonly found, an interfacial film around water

Azlinda Azizi

azlinda.azizi@research.uwa.edu.au

1 Fluid Science and Resources Division, School of Mechanical and Chemical Engineering, University of Western Australia, 35 Stirling Highway, Crawley, WA 6009, Australia

2 Department of Petroleum Engineering, Universiti Teknologi PETRONAS, 32610 Seri Iskandar, Perak Darul Ridzuan, Malaysia droplets can be formed by natural surface-active agents present in the crude oil (e.g. asphaltenes, resins and organic acids). In addition, during offshore petroleum production, the high-pressure and low-temperature conditions favour hydrate formation to occur in such W/O emulsions, which could induce large pressure drops and even pipeline flow blockage. This hydrate formation is an interfacial phenomenon that takes place when natural gas components dissolved in the oil phase comes into contact with the water phase (Sloan et al. 2010).

In order to prevent hydrate formation, effective hydrate management strategies during crude oil transportation are widely applied and researched. Traditionally, either thermodynamic hydrate inhibitors, such as methanol and monoethylene glycol (MEG), or insulating the system to remain outside of the hydrate region is employed (Frostman 2000). Thermodynamic hydrate inhibitors shift the hydrate formation curve to a higher-pressure and lower-temperature 
zone. However, this method can require large injection rates; (0.25-1 bbl methanol per bbl water produced for deep water systems) which can lead to both high capital expenditure (CAPEX) and operational expenditure (OPEX) costs. Pipeline insulation techniques can have lower OPEX, but can lead to even higher CAPEX as thermodynamic hydrate inhibitors are still required during extended shut-ins and cold well start-ups (Frostman 2000).

Hydrate anti-agglomerants (AAs) are categorised as low-dosage hydrate inhibitor (LDHI) and can be considered as alternatives to these traditional techniques. The active molecule of AAs typically contains a long hydrophobic segment that is believed to prevent the agglomeration of particles and a hydrophilic segment that interacts with the surface of the hydrate particle (Kelland 2006; Sloan and Koh 2008; Sun and Firoozabadi 2013). Numerous studies (Kelland 2006, 2009) have attributed the observed functionality of AA's to this 'hydrate-philicity'. In order to have a better understanding of the mechanisms involving AA use, Fig. 1 provides a schematic summary of the hydrate blockage formation process in oil-dominant pipelines. AAs allow the suspension of water droplets and hydrate particles (following conversion) to flow in the oil or condensate without aggregation (Sloan et al. 2010). In this way, AAs prevent the residual water phase from forming new capillary bridges between the hydrate particles; thus, the particles do not attract one another and are transported with the produced fluids at a comparatively much lower viscosity. The advantages of the use of AAs in industry include lower capital costs, lower chemical utilisation, lower transportation and storage costs because AAs can be recycled, smaller pumps due to low viscosity with the addition of AAs can lead to less maintenance costs and a hydrate control strategy which is also effective both at severe hydrate forming conditions and during extended shut-in periods (Frostman 2000). Several previous studies have reported the application of AAs to prevent hydrate plug in the field as successful and it is now a well-established technology (e.g. (Frostman 2000; Frostman et al. 2001; Frostman and Przybylinski 2001; Mehta et al. 2002)).

The stability of water-in-crude oil emulsion results from the tendency for dispersed water droplets to resist coalescence (Chan and Tao 2005). The droplet size distribution is an important emulsion characteristic influencing a range of emulsion properties including its rheology (Aichele et al. 2016; Omer and Pal 2010; Pal 1993, 2007, 2011). Hence, measurement of these W/O emulsion droplet sizes as a function of AA enables a better understanding of the impact of their addition on the flow behaviour of the system. There are several different techniques for measuring the emulsion water droplet size: nuclear magnetic resonance (NMR) pulsed field gradient (PFG) techniques, laser light diffraction and scattering, electric sensing, acoustic spectroscopy, dielectric spectroscopy, centrifugal sedimentation and optical and electron microscopy (Pavletta et al. 2004). Most are, however, not suitable for opaque concentrated emulsions as is the case for the systems studied here. The use of pulsed field gradient nuclear magnetic resonance (PFG NMR) to measure the droplet size distributions (DSDs) of emulsions is, however, readily applied to such concentrated opaque emulsions. In addition, no sample preparation (such as dilution as is required by optical microscopy) is required (Lingwood et al. 2012) and the technique is readily applicable to comparatively large and hence representative sample sizes. The aims of the work presented here are to apply these PFG NMR techniques to monitor the effect of three different industrially available hydrate AAs on W/O emulsion stability (assessed via monitoring the temporal evolution in droplet size distributions) as a complement to traditional bottle stability tests.
Fig. 1 Conceptual mechanism of hydrate blockage formation in pipelines during hydrocarbon transportation. Adapted from Turner et al. (2009) and (Aman et al. 2014, 2015)

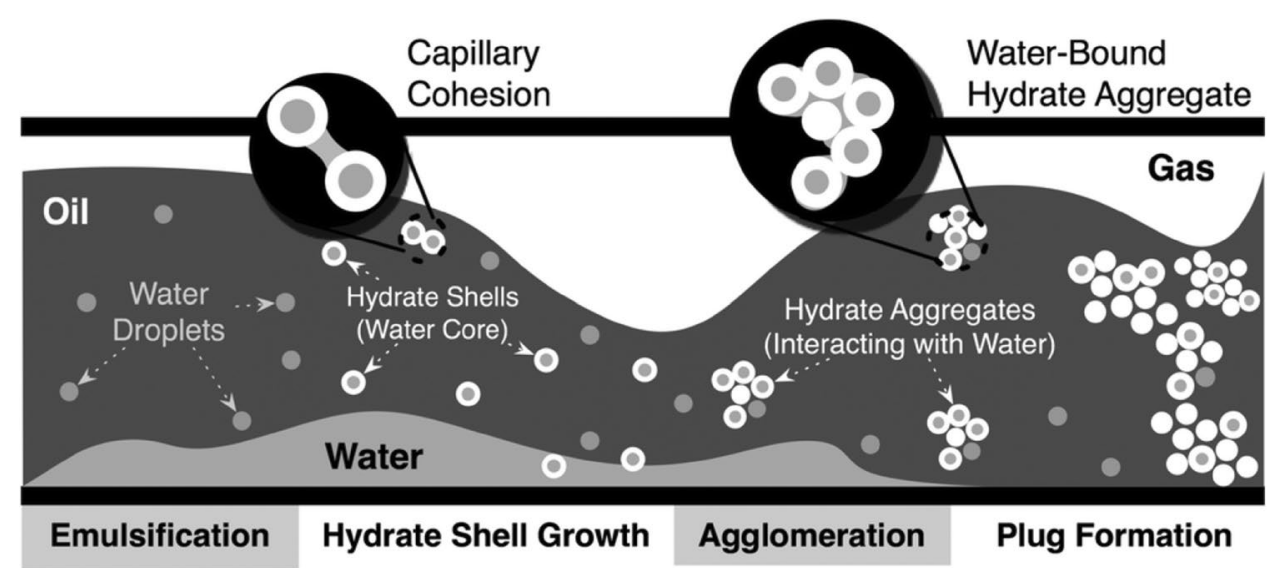




\section{Background}

\section{Relevant emulsion theory}

Emulsions are metastable suspensions consisting of discrete dispersion of droplets within an immiscible continuous phase and are typically stabilised by surface-active agents at the interface between the two phases. The surface-active agent forms an interfacial film that surrounds the droplets typically reducing the interfacial tension and rendering the system metastable. There are two main types of emulsions: oil-in-water $(\mathrm{O} / \mathrm{W})$ and water-in-oil (W/O). With respect to oilfield systems, typical water cuts and natural surfactants present result in the formation of waterin-oil emulsions (Ling et al. 2014). Less frequent, but relevant to the work presented here, are multiple emulsions in which droplets of one fluid are dispersed in larger droplets of a second fluid (Sharma et al. 2014). Multiple emulsions may be of the 'oil-in-water-in-oil' $(\mathrm{O} / \mathrm{W} / \mathrm{O})$ type or the reverse thereof $(\mathrm{W} / \mathrm{O} / \mathrm{W})$.

Surface-active agents, or surfactants, are molecules or solids that function to typically lower the interfacial tension between two phases. Conventionally surfactants are molecules with hydrophilic and hydrophobic moieties, allowing them to concentrate at the oil/water interface, stabilising the emulsion droplets and reducing the energy required to form emulsions (Salager 1994). In crude oil, these surface-active agents are, however, interfacially active species such as asphaltenes, resins, clay, silica and organic acids. The degree of emulsion stability is affected by the collective adsorption of these macromolecules and/ or fine particles at the interface (Fan et al. 2009; Wasan and Nikolov 2001). Demulsifiers act by also adsorbing at the water-oil interface in a manner that results in disruption of these surface-active agents, for example by disruption of asphaltene-resin networks that form on the droplet surface or in fact their partial removal (Delgado-Linares et al. 2016). If the interfacial film is ruptured, coalescence will occur between two contacting droplets (AcevedoMalave 2016; Pawar et al. 2011; Schoolenberg et al. 1998). If water-in-crude oil emulsions do not coalesce and phase separate in a given time frame (e.g. resident time in a primary separator), the emulsion is considered tight or stable (Alvarado et al. 2011; Angle 2001). This separation rate is also a function of the continuous oil phase viscosity (Zhang et al. 2016).

Droplet size distributions (DSDs), in particular timeresolved measurements of the DSD, play an important role in characterising emulsions. The droplet size influences many emulsions characteristics, such as the rheology (Asano and Sotoyama 1999; Otsubo and Prud'homme 1994), the long-term stability of an emulsion (Basheva et al. 1999; Ghosh et al. 2015; Kowalska et al. 2017; Tcholakova et al. 2004), emulsion liquid membrane performance (McClements and Decker 2000; Ng et al. 2010), degradation rates and resistance to creaming (Mason et al. 1996; McClements and Decker 2000), texture and optical appearance (Fernandez et al. 2004), physiological efficiency (Mason et al. 1996; McClements and Decker 2000), chemical reactivity and the kinetics of polymerisation reactions (Sood and Awasthi 2003).

\section{NMR theory}

Tanner and Stejskal (1968) first demonstrated the measurement of unrestricted self-diffusion of molecules in a fluid by PFG NMR. The method essentially relies on NMR signal attenuation due to random motion (diffusion) of the molecules between two imposed magnetic field gradients (Ling et al. 2014). The acquired NMR signal, $S$, can be related to the free self-diffusion of molecules (e.g. water in the work presented here), $D$, via the following equation (Tanner and Stejskal 1968):

$\frac{S}{S_{0}}=\exp \left[-D(\gamma g \delta)^{2}\left(\Delta-\frac{\delta}{3}\right)\right]$,

where $S_{0}$ is the signal measured when $g=0, g$ is the magnetic field gradient, $\delta$ is the duration of the magnetic field gradient applied, $\gamma$ is the gyromagnetic ratio of the nucleus of interest (in our case exclusively ${ }^{1} \mathrm{H}\left(\gamma_{1 \mathrm{H}}=2.68 \times 10^{8} \mathrm{~T}^{-1}\right.$ $\left.\mathrm{s}^{-1}\right)$ ) and $\Delta$ is the time interval between two gradient pulses. Hence, by varying $g$ while measuring $S, D$ can be extracted by regression of Eq. 1 .

In the case of restricted diffusion inside the spherical geometry of water droplet in oil, the NMR signal attenuation $(I=S(g) / S(g=0))$ can be approximated as follows (Murday and Cotts 1968):

$\ln I(D, a, g, \delta)=-2 \gamma^{2} g^{2} \sum_{m=1}^{\infty} \frac{1}{\alpha_{m}^{2}\left(\alpha_{m}^{2} a^{2}-2\right)}\left[\frac{2 \delta}{\alpha_{m}^{2} D}-\frac{\Psi}{\left(\alpha_{m}^{2} D\right)^{2}}\right]$

$\Psi=2+\exp ^{-\alpha_{m}^{2} D(\Delta-\delta)}-2 \exp ^{-\alpha_{m}^{2} D \Delta}-2 \exp ^{-\alpha_{m}^{2} D \delta}+\exp ^{-\alpha_{m}^{2} D(\Delta+\delta)}$,

where $a$ is the droplet radius and $\alpha_{\mathrm{m}}$ is the $m$ th positive root of the following equation:

$J_{\frac{5}{2}}(a \alpha)-\frac{1}{a \alpha} J_{\frac{3}{2}}(a \alpha)=0$,

where $J_{k}$ is the Bessel function of the first kind of order $k$. Equations (2a)-(2c) assume a Gaussian shape for the NMR signal phase distribution (known as the Gaussian phase distribution (gpd) model). The need to eliminate the assumption of a Gaussian phase distribution in the NMR signal

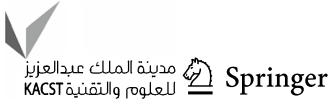


led to the development and adaptation of the block gradient pulse (bgp) method for emulsion droplet sizing. (Lingwood et al. 2012) determined that for the case of restricted diffusion inside spherical droplets, the block gradient pulse (bgp) approximation method is more accurate, where the diffusion is restricted within a spherical geometry and the consequential evolution in magnetisation is modelled using an arbitrary magnetic field based on the general gradient waveform set of methods. The bgp method is detailed elsewhere along with several successful applications to various emulsion systems (Fridjonsson et al. 2014; Haber et al. 2015; Ling et al. 2014, 2016a, b); this data analysis method is employed in this work.

Both Eq. 2 and the bgp method, however, are valid only for an emulsion with a single droplet size. For a distribution of droplet radii, $P(a)$, the measured NMR signal is given by the following integral:

$\frac{S}{S_{0}}=I(D, a, g, \delta)=\frac{\int_{0}^{\infty} a^{3} P(a) I(D, a, g, \delta) \mathrm{d} a}{\int_{0}^{\infty} a^{3} P(a) \mathrm{d} a}$,

where $I(D, a, g, \delta)$ is the signal attenuation function-as detailed above this is the bgp method in this work. Tikhonov regularisation has been applied to invert Eq. 3 numerically (Hollingsworth and Johns 2003) and has been successfully demonstrated on a wide range of emulsions in previous work (Fridjonsson et al. 2014; Haber et al. 2015; Ling et al. 2014, 2016a).

\section{Methodology}

In this study, we consider water-in-oil emulsions formulated from a local West Australian crude oil blend of 'light' and 'heavy' crude oil as follows: 95\% 'light:5\% 'heavy'. (The resultant density is $0.88 \mathrm{~g} / \mathrm{mL}$; the resultant viscosity is $31 \mathrm{mPa} \mathrm{s}$ at $25^{\circ} \mathrm{C}$.) A W/O emulsion was formed using this crude oil blend and $30 \mathrm{wt} \%$ deionised (DI) water. Each emulsion sample was prepared using a $40 \mathrm{~g}$ batch of oil and was sheared at $17,500 \mathrm{rpm}$ for $5 \mathrm{~min}$ using a high-speed MICCRA D-9 Homogenizer manufactured by ART Prozess \& Labortechnik, GmbH. While the oil is being homogenised, the water that has been mixed with the relevant AA chemical was added drop-wise to the centre of the oil phase. The selected (current generation) AAs from multiple industrial sources were pre-mixed and dissolved at $2 \mathrm{wt} \%$ in the aqueous phase prior to homogenisation. A water-in-crude oil emulsion without any chemicals was also prepared as a reference emulsion.

\section{Bottle stability tests}

After homogenisation in 100-ml Schott bottles, the emulsion systems were monitored visually over the course of $10 \mathrm{~h}$, to quantify the volume of the residual emulsion layer relative to the total liquid volume in the sample (hereafter simply referred to as the percentage of emulsion). The position of the oil/emulsion and/or oil/water interface as schematically shown in Fig. 2 was observed as an indication of phase separation and recorded every $1 \mathrm{~h}$.

Percentage of emulsion versus time was then plotted; this is very simply defined as (Hajivand and Vaziri 2015):

$\left(\% \frac{V}{V}\right)=\frac{V_{1}}{V_{2}} \times 100$,

where $V_{1}$ is the volume of remaining emulsion after water and oil separation and $V_{2}$ is the original volume of the initial emulsion. The resultant fraction is thus bounded between 0 and $100 \%$, where $0 \%$ indicates complete destabilisation of the emulsion and $100 \%$ nominally indicates complete stability.

\section{Droplet size distribution measurement}

After each homogenisation stage, a small subsample of the emulsion layer was rapidly transferred to three different NMR sample tubes to characterise the droplet size distribution using a mobile Magritek low-field NMR system. (Different glass pipettes were used to carefully extract and transfer the samples from three different layers of emulsion to avoid contamination and mixing between the different layers.) The main NMR hardware used for this study was a bench-top $1 \mathrm{~T}$ permanent magnet featuring a Halbach magnet array and a 5-mm-inner-diameter rf coil tuned to the ${ }^{1} \mathrm{H}$ resonance of $40 \mathrm{MHz}$; the measurements were all performed at $23^{\circ} \mathrm{C}$. This magnet arrangement provides a sufficiently homogeneous magnetic field (water resonance

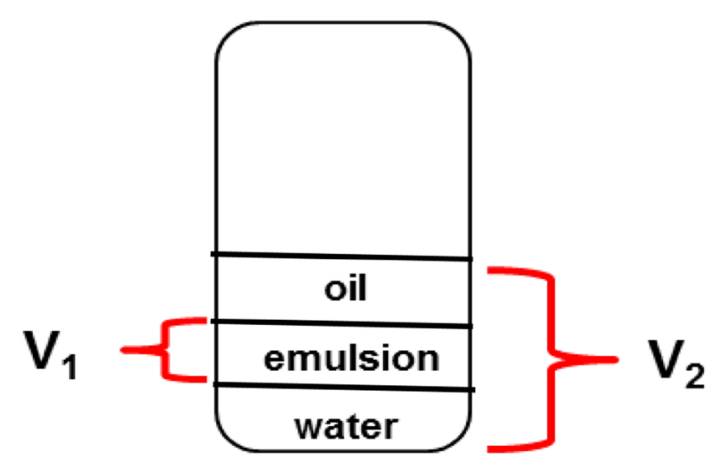

Fig. 2 Schematic diagram for measurement of percentage of emulsion after separation 
Fig. 3 Stimulated echo pulsed field gradient (STE PFG) pulse sequence used for diffusion measurements
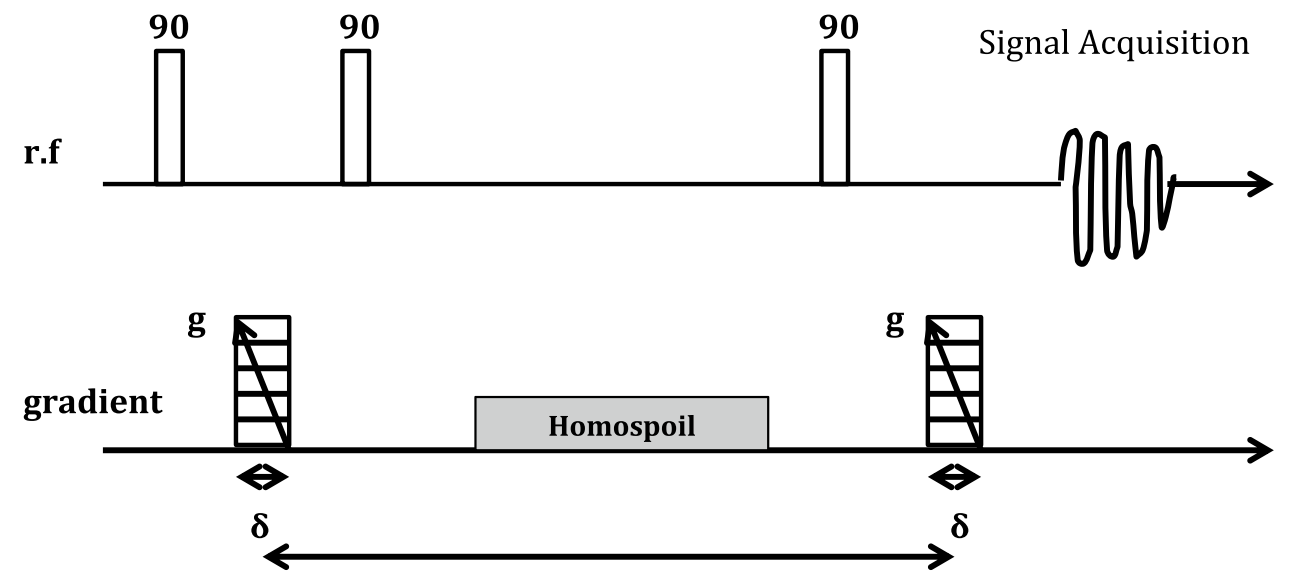

$\Delta$

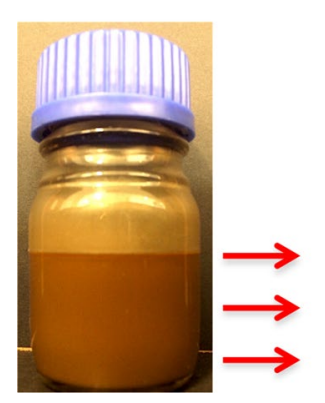

\section{Top Middle Bottom}

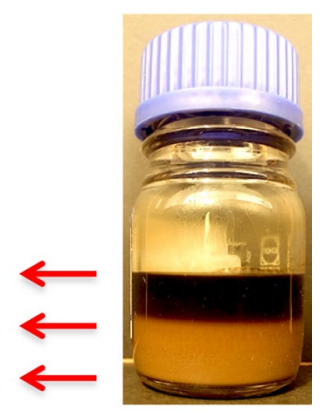

Fig. 4 Top, middle and bottom physical locations where samples were taken for diffusion measurements and hence droplet sizing using NMR. An oil layer is observed to form for the emulsion of the right

peak width of $\sim 4 \mathrm{~Hz}$ at half maximum) such that chemical shift resolution of the oil and water NMR signal is readily achieved. Via the analysis of the signal peak in the acquired spectra corresponding to the chemical shift of water, an unambiguous analysis of the water phase diffusion is performed. A custom-built gradient coil, of maximum strength $1 \mathrm{~T} / \mathrm{m}$, was used for all required pulsed field gradient diffusion experiments. The magnet/coil assembly is driven by a Kea2 spectrometer using the software package, Prospa. All pulsed field gradient (PFG) measurements were taken using the stimulated echo pulsed field gradient sequence (STE PFG) as shown in Fig. 3.

For DSD measurements in this study, the required NMR data were captured with a repetition time (RT) between signal acquisitions $=9000 \mathrm{~ms}, \delta=4 \mathrm{~ms}$ and $\Delta=200 \mathrm{~ms}$ and with the maximum gradient strength $g=1 \mathrm{~T} / \mathrm{m}$. The DSD measurements using PFG NMR were taken using samples taken from three constant physical locations: top, middle and bottom of the Schott bottles as shown in Fig. 4 for every hour up to $10 \mathrm{~h}$. (Different glass pipettes were used when transferring each emulsion sample to NMR sample tube to avoid contamination.) These were performed in order to investigate the evolution of water droplet size as the emulsion coalesced and settled; the layers above and below the emulsions gradually separated into water and oil phases over time.

\section{Results and discussions}

\section{Emulsion stability}

For bottle stability tests, the percentages of emulsion for all samples were quantified based on changes in emulsion thickness in the bottle every hour. Figure 5 shows that no significant change in the percentage of emulsions in $10 \mathrm{~h}$ for emulsions formulated with the addition of AA- 1 and AA-2, respectively. Meanwhile, Fig. 6 shows the photographs of emulsion taken during the $10 \mathrm{~h}$ bottle stability test. This indicates that these industrial AAs likely have the ability to reduce the interfacial tension at the interface between water and oil and make the emulsions more stable. AA-3 has, in contrast, demulsified the emulsions and separated it into significant oil and water phases over the $10 \mathrm{~h}$ period.

\section{Droplet size distributions}

Emulsions with current industrial AAs were characterised using low-field NMR, as described in the "Droplet size distribution measurement" section, and showed significantly different behaviours over $10 \mathrm{~h}$. Figures 7, 8, 9 and 10 show the resultant emulsion DSDs for the reference (blank) waterin-oil emulsion and water-in-oil emulsion with the addition of AA-1, AA-2 and AA-3, respectively, measured at three different physical locations: top, middle and bottom of the Schott bottle sample. The corresponding mean droplet sizes for all measurements taken are summarised in Fig. 11. From the DSDs in Fig. 7, the blank emulsion system remained reasonably stable over the $10 \mathrm{~h}$ observation period for those

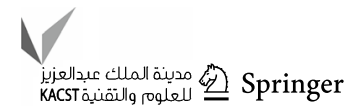


Fig. 5 Percentage of emulsion as a function of time for three AA emulsion systems (blue) and the reference (blank) emulsion (black). Each data point and associated error bar represent the mean and standard deviation resulting from independent triplicate measurements

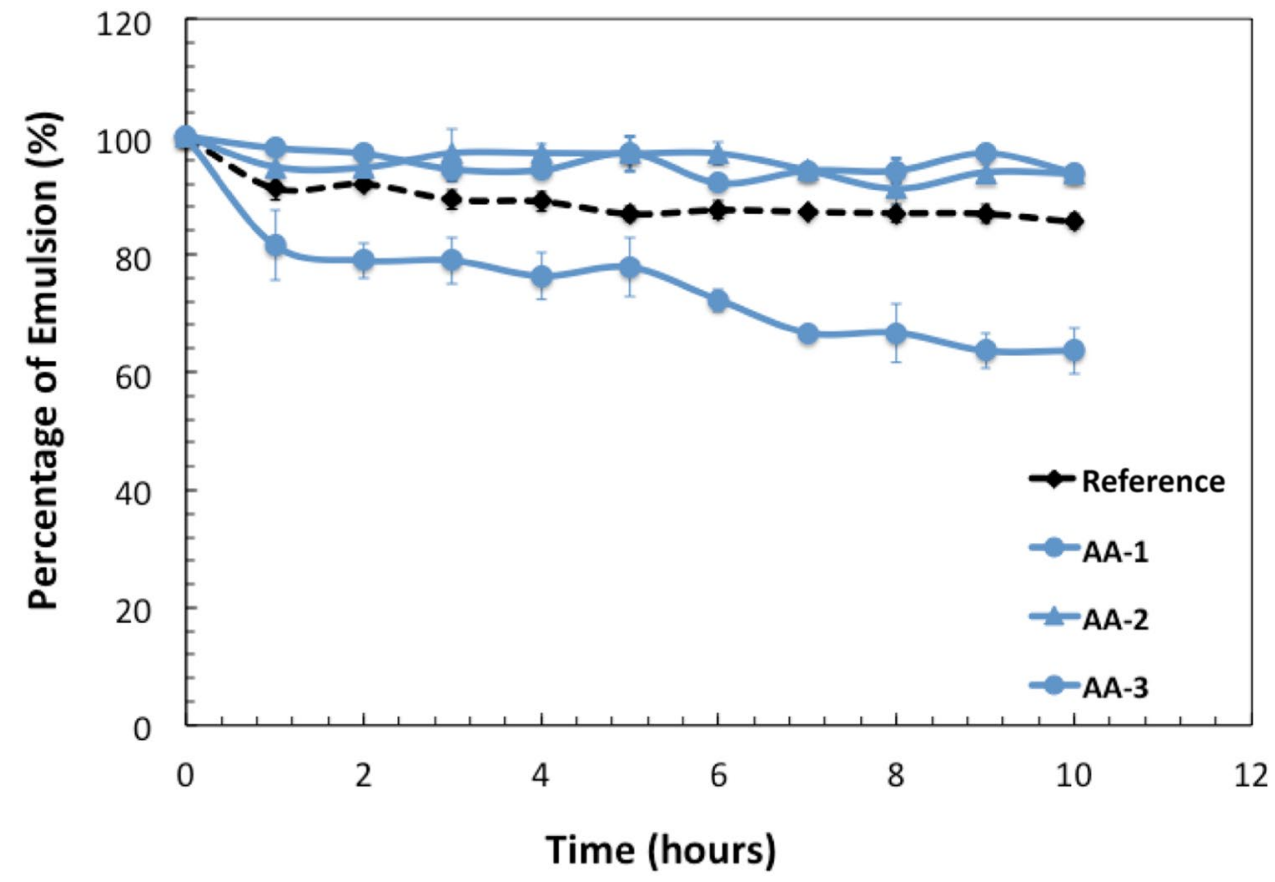

Fig. 6 Photographs for emulsion in 10-hour observation for the reference (blank) sample and with the presence of AA-1, AA-2 and AA-3

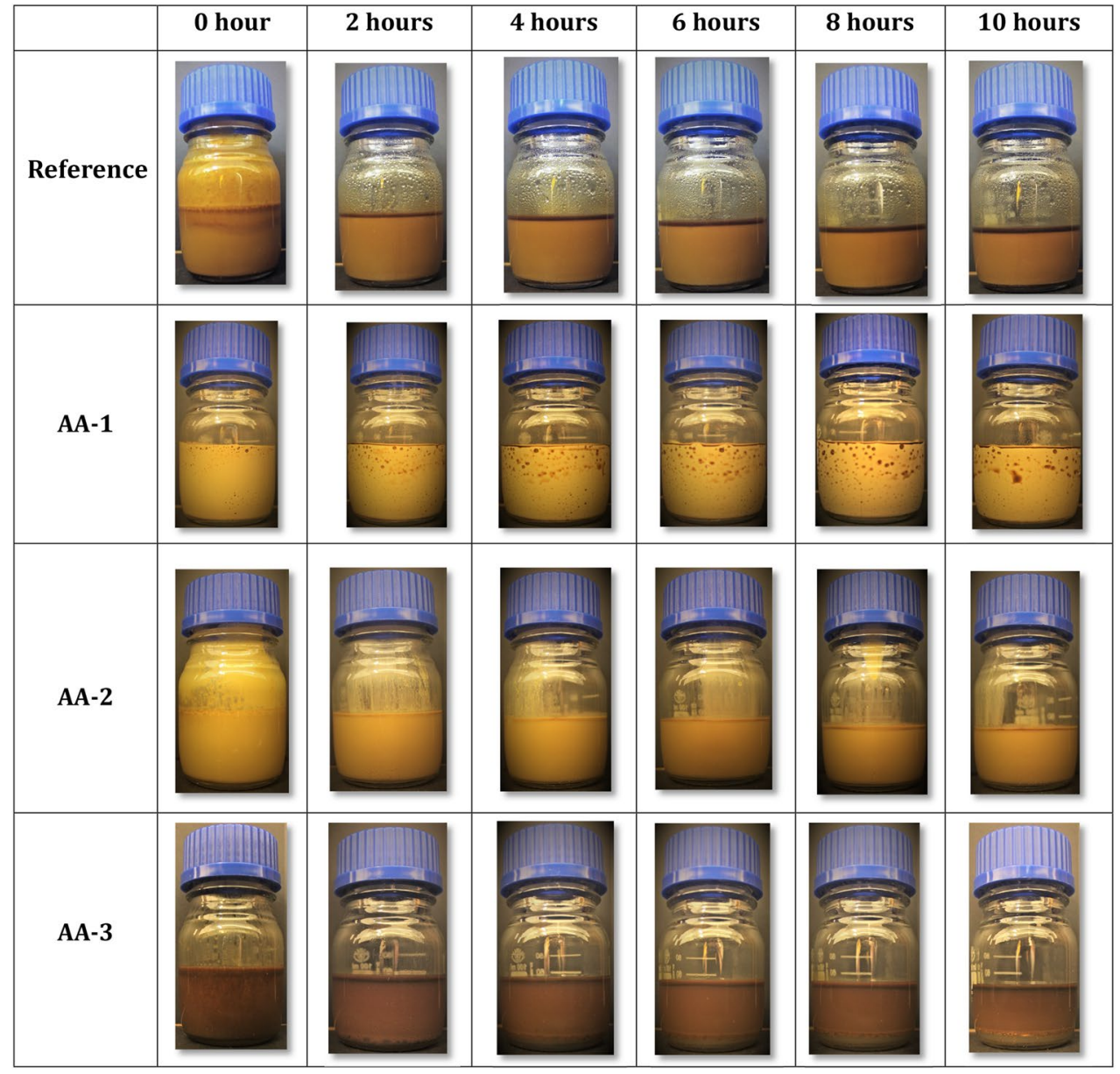




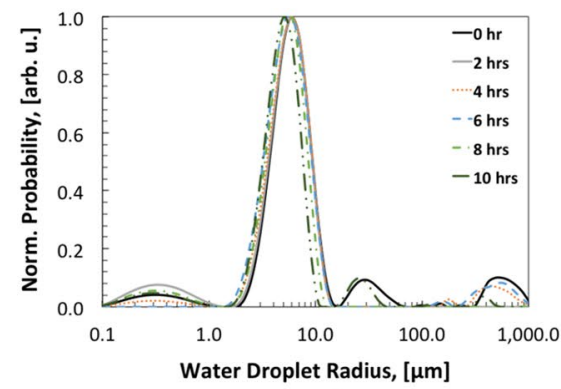

(a)

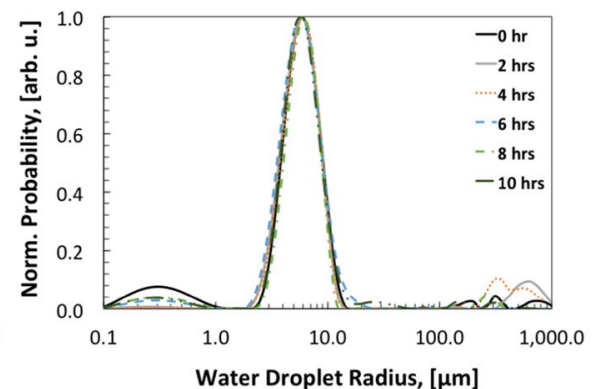

(b)

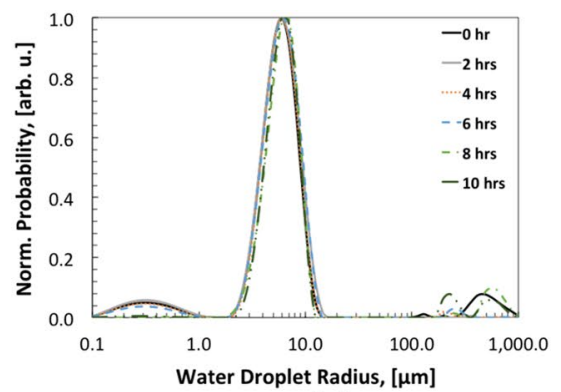

(c)
Fig. 7 Droplet size distributions for: a top, $\mathbf{b}$ middle and $\mathbf{c}$ bottom physical locations of the reference (blank) water-in-oil emulsion obtained from the PFG NMR diffusion experiments. This blank sample is used as a reference. No significant shift for the middle and bottom peaks exhibits stable emulsion over $10 \mathrm{~h}$. The peaks at sized larger than 100 microns reflect system noise and effectively limited acquisition bandwidth

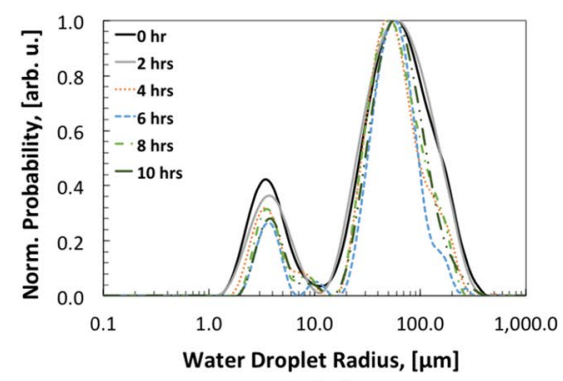

(a)

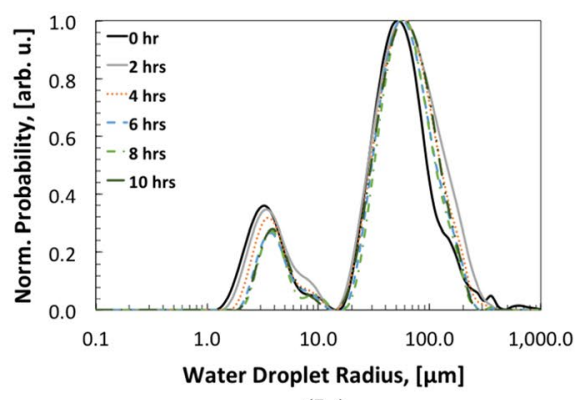

(b)

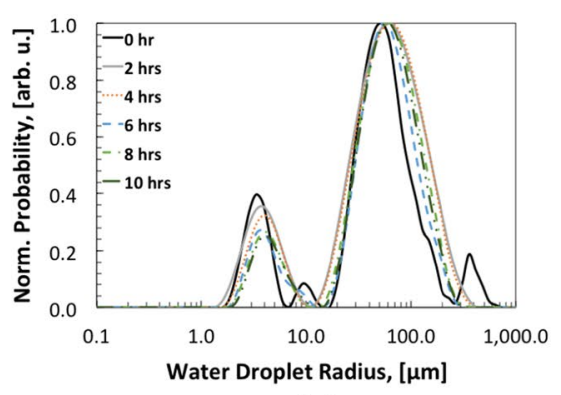

(c)

Fig. 8 Droplet size distributions for: a top, $\mathbf{b}$ middle and $\mathbf{c}$ bottom physical locations of water-in-oil emulsion formulated with AA-1 obtained from the PFG NMR diffusion experiments. Two populations of water represent small droplets and relatively free water phase

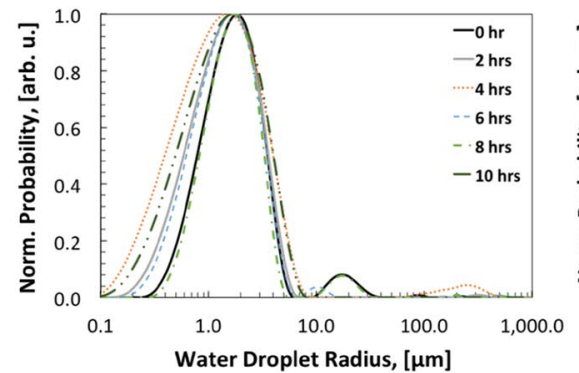

(a)

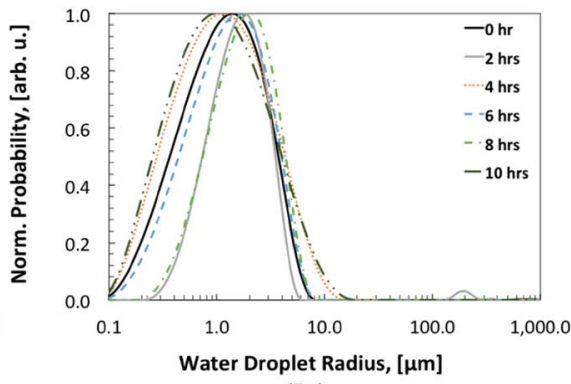

(b)

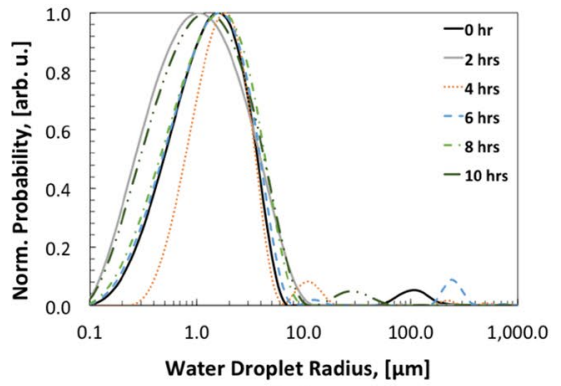

(c)

Fig. 9 Droplet size distributions for: a top, $\mathbf{b}$ middle and $\mathbf{c}$ bottom physical locations of water-in-oil emulsion formulated with AA-2 obtained from the PFG NMR diffusion experiments. This AA has resulted in much smaller droplets compared to the blank emulsion

three different physical locations. The mean water droplet size data presented in Fig. 11a show that all three physical locations quantitatively reveal virtually no change over $10 \mathrm{~h}$.

Note that the W/O emulsion formulated with AA-1 shows marginally greater emulsion stability compared to the blank sample W/O emulsion (evident in Figs. 5 and 6). However, as observed in Fig. 11b, the resultant emulsion contains much larger droplets relative to the blank emulsion. Thus, in this comparative case, greater emulsion stability is not a consequence of greater surfactant adsorption at the droplet interface resulting in smaller droplets. According to (Delgado-Linares et al. 2016), a mixture of surfactants has been demonstrated to stabilise the emulsions more efficiently than a single surfactant, owing to synergetic mechanisms that 


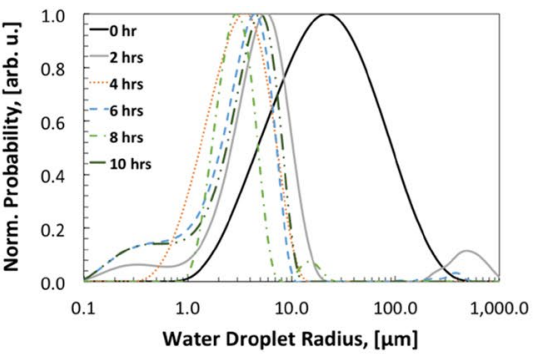

(a)

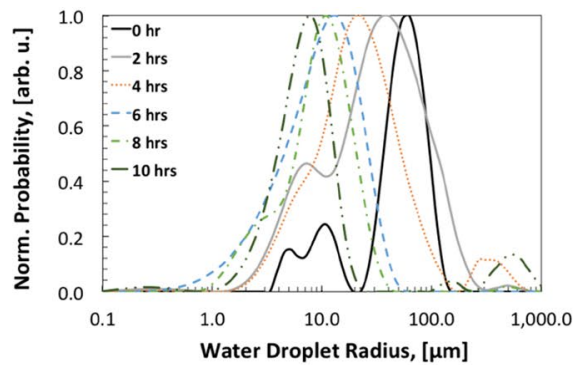

(b)

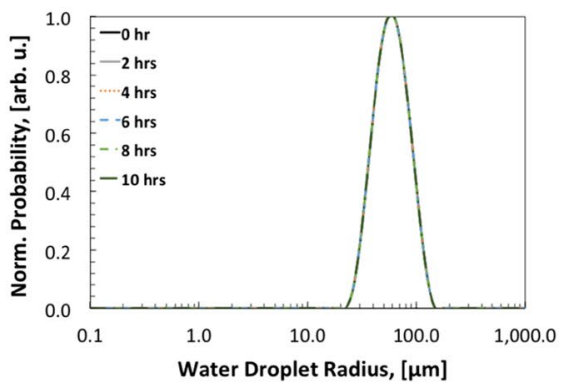

(c)

Fig. 10 Droplet size distributions for: $\mathbf{a}$ top, $\mathbf{b}$ middle and $\mathbf{c}$ bottom physical locations of water-in-oil emulsion formulated with AA-3 as obtained from the PFG NMR diffusion experiments

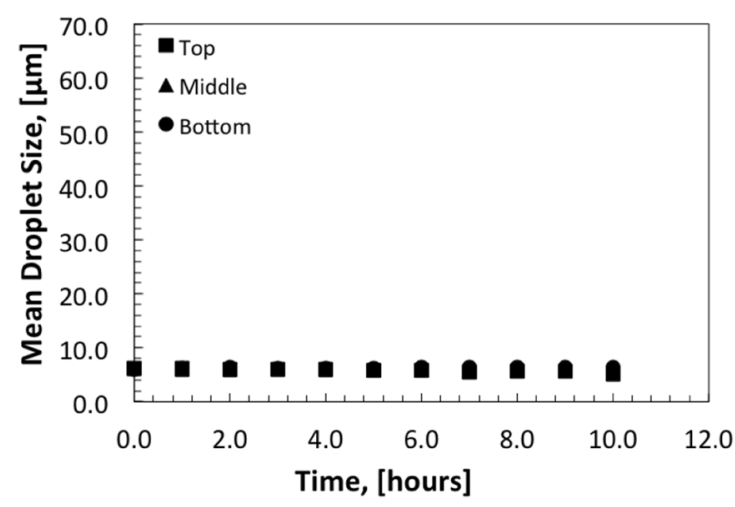

(a)

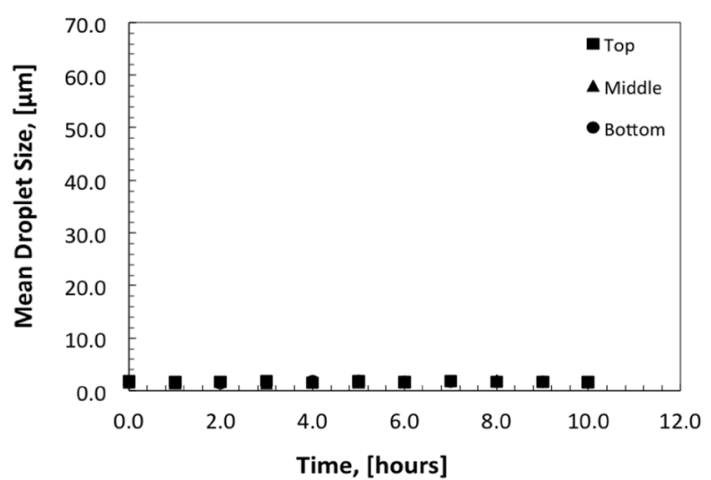

(c)

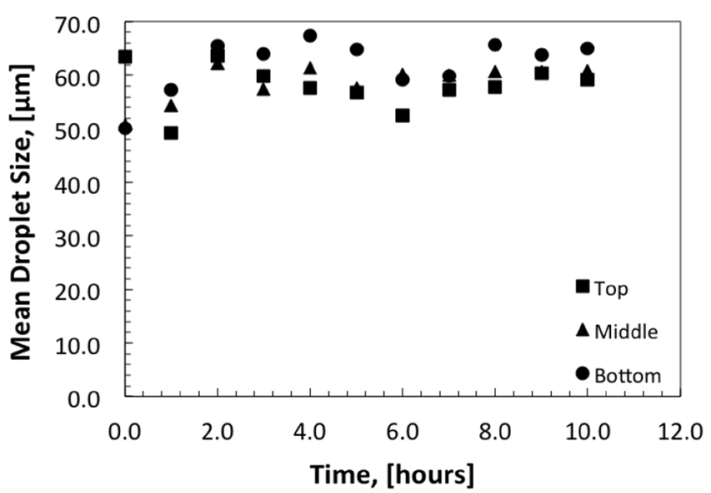

(b)

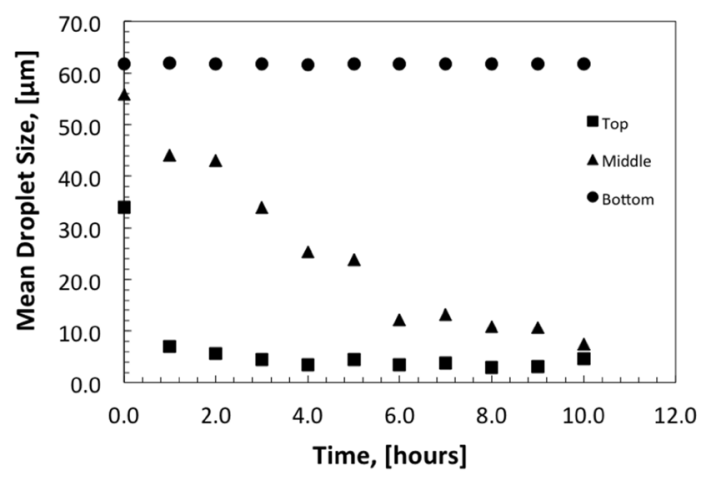

(d)

Fig. 11 Mean water droplet sizes for: a blank water-in-oil emulsion sample and water-in-oil emulsion with b AA-1, c AA-2 and d AA-3, respectively

reduce the coalescence of droplets. However, in this case the two distinct droplet populations, both of which do not change with either position or time as shown in Fig. 8, are indicative of the formation of a multiple emulsion (Hughes et al. 2013) in the form of a water-in-oil-in-water emulsion. Such emulsion microstructures are encouraged by the presence of different surfactants in the mixture that stabilise water and oil droplets, respectively (Neumann et al. 2018). It is interesting that this was sufficient to form such a structure upon simple shear application and sample mixing; normally, multiple emulsions require the sequential addition of the surfactants and the initial formation of the inner droplets. The formation of this W/O/W emulsion was confirmed using simple conductivity measurements, which indicated nonzero conductivity and thus a continuous water phase. 
More conventional behaviour is observed in Fig. 9, where AA-2 is observed to act as a conventional emulsifier that results in much smaller droplets (approximately a factor of 5-evident in Fig. 11a, c) than the blank emulsion. In this case, clearly AA-2 is reducing the interfacial tension of the system resulting in much smaller droplets. The DSDs are also well described by a log-normal shape which generally indicates emulsions that have been sheared to their minimum size.

In contrast in Fig. 10, the addition of AA-3 has resulted in not only a visually less stable emulsion but one in which the PFG NMR measurements of emulsion DSD show significant change over the 10-h period. The bottom of the sample persists with larger droplets that are indicative of the formation of a substantial free water layer in this zone. As shown in Fig. 11d, the top of the sample shows a rapid decline in mean droplet size, a process that is substantially slower in the middle of the sample. This clearly indicates the gradual passage of larger water droplets to the bottom of the sample (settling) as the emulsion is destabilised. Only smaller water droplets subsequently remained in these regions, and the much poorer signal-to-noise ratio (SNR) for measurements in these regions as time progressed indicated a significant reduction in their water content. Collectively, the results demonstrated that AA-3 acted as a demulsifier in the system tested.

\section{Conclusions}

This study demonstrates that the combination of bottle stability tests and PFG NMR measurements to obtain emulsion DSDs is useful diagnostic tools to explore emulsion stability. Here, the method was employed to quantify the evolution in emulsion DSDs and macroscopic emulsion stability with and without industrial AAs. The results demonstrated quite different behaviours for different AAs. Relative to the blank emulsion, the AAs were observed to both destabilise and stabilise the W/O emulsion. There was also evidence that one of the AAs resulted in the formation of a W/O/W multiple emulsions that was extremely stable. The formation of both different emulsion microstructures and different emulsion stabilities depending on industrial AA selection has significant implications for both emulsion transport in pipelines and any hydrate formation that occurs.

Open Access This article is distributed under the terms of the Creative Commons Attribution 4.0 International License (http://creativeco mmons.org/licenses/by/4.0/), which permits unrestricted use, distribution, and reproduction in any medium, provided you give appropriate credit to the original author(s) and the source, provide a link to the Creative Commons license, and indicate if changes were made.

\section{References}

Acevedo-Malave A (2016) Fluid mechanics calculations in physics of droplets II: a study of head-on collisions for the system waterheptane. J Comput Multiphase Flows 8:109-119

Aichele CP, Raman AKY, Venkataramani D, Bhagwat S, Martin T, Clark PE (2016) Emulsion stability of surfactant and solid stabilized water-in-oil emulsions after hydrate formation and dissociation. Colloids Surf A 506:607-621

Alvarado V, Wang X, Moradi M (2011) Stability proxies for waterin-oil emulsions and implications in aqueous-based enhanced oil recovery. Energies 4:1058-1086

Aman ZM, Pfeiffer K, Vogt SJ, Johns ML, May EF (2014) Corrosion inhibitor interaction at hydrate-oil interfaces from differential scanning calorimetry measurements. Colloids Surf A 448:81-87

Aman ZM, Haber A, Ling NNA, Thornton A, Johns ML, May EF (2015) Effect of brine salinity on the stability of hydrate-in-oil dispersions and water-in-oil emulsions. Energy Fuels 29:7948-7955

Angle CW (2001) Chemical demulsification of stable crude oil and bitumen emulsions in petroleum recovery-a review. In: Sjoblom $\mathrm{J}$ (ed) Encyclopedic handbook of emulsion technology. Marcel Dekker, New York, pp 541-594

Asano Y, Sotoyama K (1999) Viscosity change in oil/water food emulsions prepared using a membrane emulsification system. Food Chem 66:327-331

Basheva ES, Gurkov TD, Ivanov IB, Bantchev GB, Campbel B, Borwankar RP (1999) Size dependence of the stability of emulsion drops pressed against a large interface. Langmuir 15:6764-6769

Chan G, Tao D (2005) An experimental study of stability of oil in water emulsion. Fuel Process Technol 86:499-508

Delgado-Linares JG, Alvarado JG, Véjar F, Bullón J, Forgiarini AM, Salager J-L (2016) Breaking of water-in-crude oil emulsions. 7. Demulsifier performance at optimum formulation for various extended surfactant structures. Energy Fuels 30:7065-7073. https ://doi.org/10.1021/acs.energyfuels.6b01286

Fan Y, Simon S, Sjoblom J (2009) Chemical destabilization of crude oil emulsions: effect of nonionic surfactants as emulsion inhibitors. Energy Fuels 23:4575-4583

Fernandez P, Andre V, Rieger J, Kuhnle A (2004) Nano-emulsion formation by emulsion phase inversion. Colloid Surf A-Physicochem Eng Asp 251:53-58

Fridjonsson EO, Graham BF, Akhfash M, May EF, Johns ML (2014) Optimized droplet sizing of water-in-crude oil emulsions using nuclear magnetic resonance. Energy Fuels 28:1756-1764

Frostman LM (2000) Anti-agglomerant hydrate inhibitors for prevention of hydrate plugs in deepwater systems. In: SPE 63122

Frostman LM, Przybylinski JL (2001) Successful applications of antiagglomerant hydrate inhibitors. In: Proceedings of the society of petroleum engineers (SPE) international symposium on oilfield chemistry, Houston, TX

Frostman LM, Gallagher CG, Ramachandran S, Weispfennig K (2001) Ensuring systems compatibility for deepwater chemicals. In: Proceedings of the society of petroleum engineers (SPE) international symposium on oilfield chemistry, Houston, TX,

Ghosh S, Pradhan M, Patel T, Haj-shafiei S, Rousseau D (2015) Longterm stability of crystal-stabilized water in oil emulsions. J Colloid Interface Sci 460:247-257

Haber A, Akhfash M, Koh CA, Aman ZM, Fridjonsson EO, May EF, Johns ML (2015) Hydrate shell growth measured using NMR. Langmuir 31:8786-8794. https://doi.org/10.1021/acs.langm uir.5b01688

Hajivand P, Vaziri A (2015) Optimization of demulsifier formulation for separation of water from crude oil emulsions. Braz J Chem Eng 32:107-118. https://doi.org/10.1590/0104-6632.20150321s0 0002755 
Hollingsworth KG, Johns ML (2003) Measurement of emulsion droplet sizes using PFG NMR and regularization methods. J Colloid Interface Sci 258(2):383-389

Hughes E et al (2013) NMR analysis and microfluidic preparation of mono-disperse water-in-oil-in-water double emulsions. J Colloid Interface Sci 1:147-156

Kelland MA (2006) History of the development of low dosage hydrate inhibitors. Energy Fuels 20:825-847

Kelland MA (2009) Production chemicals for the oil and gas industry. Chromatographia 72:199

Kowalska M, Zbikowska A, Wozniak M, Kucharczyk K (2017) Long term stability of emulsion based on rose oil. J Dispers Sci Technol 38:1563-1569

Ling NAA, Haber A, May EF, Fridjonsson EO, Johns ML (2014) NMR studies of emulsion microstructure approaching the phase inversion point. Colloids Surf A 462:244-251

Ling NNA, Haber A, Fridjonsson EO, May EF, Johns ML (2016a) Shear-induced emulsion droplet diffusion studies using NMR. J Colloid Interface Sci 464:229-237

Ling NNA, Haber A, Hughes TJ, Graham BF, May EF, Fridjonsson EO, Johns ML (2016b) NMR studies of the effect of $\mathrm{CO}_{2}$ on oilfield emulsion stability. Energy Fuels 30:5555-5562. https://doi. org/10.1021/acs.energyfuels.6b00782

Lingwood IA, Chandrasekera TC, Kolz J, Fridjonsson EO, Johns ML (2012) Emulsion droplet sizing using low-field NMR with chemical shift resolution and the block gradient pulse method. J Magn Reson 214:281-288

Mason TG, Krall AH, Gang H, Bibette J, Weitz DA (1996) Monodisperse emulsions: properties and uses encyclopedia of emulsion technology. Marcel Dekker, New York

McClements DJ, Decker EA (2000) Lipid oxidation in oil-in-water emulsions: impact of molecular environment on chemical reactions in heterogeneous food systems. J Food Sci 65:1270-1282

Mehta AP, Herbert PB, Cadena ER, Weatherman JP (2002) Successful applications of anti-agglomerant hydrate inhibitors. In: Proceedings of the offshore technology conference, Houston, TX

Murday JS, Cotts RM (1968) Self-diffusion coefficient of liquid lithium. J Chem Phys 48:4938-4945

Neumann SM, Wittstock N, Schaaf USVD, Karbstein HP (2018) Interactions in water in oil in water double emulsions: systematical investigations on the interfacial properties and emulsion structure of the outer oil in water emulsion. Colloids Surf A 537:524-531

Ng YS, Jayakumar NS, Hashim MA (2010) Performance evaluation of organic emulsion liquid membrane on phenol removal. J Hazard Mater 184:255-260

Omer A, Pal R (2010) Pipeline flow behavior of water-in-oil emulsions with and without a polymeric additive in the aqueous phase. Chem Eng Technol 33:983-992

Otsubo RY, Prud'homme K (1994) Rheology of oil-in-water emulsions. Rheol Acta 33:29-37
Pal R (1993) Pipeline flow of unstable and surfactant-stabilized emulsions. AIChE J39:1754-1764

Pal R (2007) Mechanism of turbulent drag reduction in emulsions and bubbly suspensions. Ind Eng Chem Res 46:618-622

Pal R (2011) Rheology of simple and multiple emulsions. Curr Opin Colloid Interface Sci 16:41-60

Pavletta SD, Slavka T, Nikolai DD, Krassimir DD, Bruce C, Catherine S, Dennis K (2004) Evaluation of the precision of drop-size determination in oil/water emulsions by low resolution NMR spectroscopy. Langmuir 20:11402-11413

Pawar AB, Caggioni M, Ergun R, Hartel RW, Spicer PT (2011) Arrested coalescence in Pickering emulsions. Soft Matter 7:7710-7716

Salager JL (1994) Interfacial phenomena in dispersed systems. Universidad De Los Andes, Mérida-Venezuela

Schoolenberg GE, During F, Ingenbleek G (1998) Coalescence and interfacial tension measurements for polymer melts: experiments on a PS-PE model system. Polymer 39:765-772

Sharma S, Shukla P, Misra A, Mishra PR (2014) Interfacial and colloidal properties of emulsified systems: pharmaceutical and biological perspective. Colloid Interface Sci Pharm Res Dev 8:149-172

Sloan D, Koh CA (2008) Clathrate hydrates of natural gases, 3rd edn. CRC Press, Boca Raton

Sloan D, Koh C, Sum KA (2010) Natural gas hydrates in flow assurance gulf professional. Gulf Professional Publishing, Burlington, MA

Sood A, Awasthi SK (2003) Initial droplet size distribution in miniemulsion polymerization. J Appl Polym Sci 88:3058-3065

Sun M, Firoozabadi A (2013) New surfactant for hydrate anti-agglomeration in hydrocarbon flowlines and seabed oil capture. J Colloid Interface Sci 402:312-319

Tanner JE, Stejskal EO (1968) Restricted self-diffusion of protons in colloidal systems by the pulsed-gradient. Spin-Echo Method J Phys Chem 49:1768-1777. https://doi.org/10.1063/1.1670306

Tcholakova S, Denkov N, Ivanov IB, Marinov R (2004) Evaluation of short term and long term stability of emulsions by centrifugation and NMR. Bulg J Phys 31:96-110

Turner D, Miller K, Sloan D (2009) Methane hydrate formation and an inward growing shell model in water-in-oil dispersions. Chem Eng Sci 64:3996-4004

Wasan DT, Nikolov AD (2001) Structure and stability of emulsions. In: Sjoblom J (ed) Encyclopedic handbook of emulsion technology. Marcel Dekker, New York, pp 59-70

Zhang J, Tian D, Lin M, Yang Z, Dong Z (2016) Effect of resins, waxes and asphaltenes on water-oil interfacial properties and emulsion stability. Colloids Surf A 507:1-6

Publisher's Note Springer Nature remains neutral with regard to jurisdictional claims in published maps and institutional affiliations. 